\title{
A Dipole Approximation of the Backscattering From a Conductor in a Semi-Infinite Dissipative Medium
}

\author{
Martin B. Kraichman \\ Contribution from U.S. Naval Ordnance Laboratory, White Oak, Silver Spring, Md.
}

(Received February 11,1963)

\begin{abstract}
The backscattering of a uniform plane wave by a conductor in a semi-infinite dissipative medium is discussed. The conductor is assumed to act as both an electric and a magnetic dipole with moments which are obtained from the electric and magnetic polarizabilities of the conductor, respectively. Using these induced moments, expressions are derived for the backscattered electric field at a point on the surface of the dissipative half-space directly above the dipoles. Both harmonic and transient excitation are considered.
\end{abstract}

\section{Introduction}

The scattering of electromagnetic waves by conductors in an infinite homogeneous dissipative medium has been treated by various investigators in recent years. The classical problem of the scattering of a plane wave by a spherical object is reviewed by Stratton [1941]. Wait [1951, 1960] has considered the response of a conducting sphere to a uniform time varying magnetic field and to the fields of electric and magnetic dipoles. The scattering by an infinite inhomogenous conducting cylinder under the influence of a time varying magnetic field has been investigated by Negi [1962].

In many practical situations, it is of interest to consider the scattering of electromagnetic waves by conductors in a semi-infinite homogeneous dissipative medium. Recently, Galejs [1962], using a dipole approximation, treated the problem of the scattering from a conducting. sphere in such a medium when the sphere is excited by a surface wave or by fields from vertical electric or horizontal magnetic dipoles in the lossless half-space. The analysis, however, is restricted to field points with horizontal ranges from the sphere which are much greater than a wavelength in the dissipative medium.

The present paper considers the scattering of a uniform plane wave by a conductor of finite dimensions embedded in a semi-infinite dissipative medium. The frequency of excitation and the conductivity of the medium are such that the displacement current can be neglected. A dipole approximation, in which the electric and magnetic dipole moments of the conductor are obtained from the electric and magnetic polarizabilities respectively, is used to evaluate the backscattered electric field in the interface directly above the conductor. The electric field step response is obtained from the response to harmonic excitation by transforming the Fourier integral into a convolution integral. Graphs of the electric field step responses are presented for the backscattering from both electric and magnetic dipoles.

\section{Dipole Approximation}

A uniform plane wave is assumed to propagate vertically in a semi-infinite dissipative medium having a horizontal planar interface. The displacement current in the dissipative medium is considered to be negligible compared with the conduction current. The upper half-space is assumed to have the properties of free space. A perfectly conducting body (or one with negligible field penetration), which is embedded in the dissipative half-space, will have a surface current induced on it by the incident plane wave. The resulting scattered electromagnetic field may be approximated by that from a horizontal electric dipole and a horizontal magnetic dipole if the vertical dimension of the conductor is much smaller than a wavelength in the dissipative medium and if the dimensions of the conductor are small compared 
with the distance to a field point. In this approximation, the electric field of the plane wave induces a horizontal electric dipole moment in the conductor while the magnetic field induces a horizontal magnetic dipole moment. Both of these moments act as a source of electromagnetic field which can be determined from the propagation equations for horizontal electric and magnetic dipoles in a semi-infinite conducting medium.

\subsection{Induced Flectric Dipole Moment}

Referring to the rectangular coordinates shown in figure 1, the surface of a semi-infinite dissipative medium is located at $z=0$. A uniform plane wave, with harmonic variation $e^{j \omega t}$, propagates in the medium in the positive $z$ direction. The electric and magnetic fields are given by $E_{x}(z, \omega)$ and $H_{y}(z, \omega)$ respectively. If the incident electric field is substantially uniform over the height ( $z$-dimension) of a perfect conductor at a depth $z=d$, the conductor will acquire an induced electric dipole moment, $P_{x}(d, \omega)$, which may be expressed as

where

$$
P_{x}(d, \omega)=\left[\epsilon\left(1+\frac{\sigma}{j \omega \epsilon}\right)\right] \alpha_{e} E_{x}(d, \omega),
$$

$$
\begin{aligned}
\epsilon & =\text { permittivity of the dissipative medium, } \\
\sigma & =\text { conductivity of the dissipative medium, } \\
\alpha_{e} & =\text { electric polarizability of the perfectly conducting body, } \\
E_{x}(d, \omega) & =\text { incident electric field at a depth } d .
\end{aligned}
$$

The quantity enclosed in brackets represents the complex permittivity of the dissipative medium. If the displacement current in the dissipative medium is neglected $(\sigma>>\omega \epsilon)$, then (1) reduces to

$$
P_{x}(d, \omega)=\frac{\sigma}{j \omega} \alpha_{e} E_{x}(d, \omega)
$$

The current moment $P_{x}^{\prime}(d, \omega)$ may be obtained by multiplying the right side of (2) by $j \omega$. Thus,

$$
P_{x}^{\prime}(d, \omega)=j \omega P_{x}(d, \omega)=\sigma \alpha_{e} E_{x}(d, \omega)
$$

and the induced electric current moment is seen to be in phase with the incident electric field. Multiple reflections between the conducting body and the surface of the half-space will be considered later in this section.

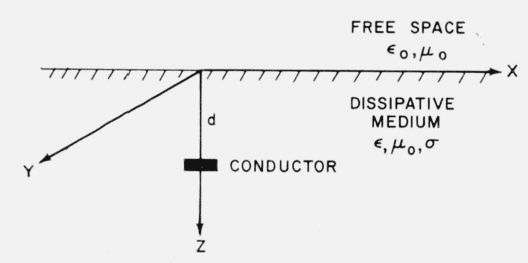

FIgURE 1. A conductor embedded in a semi-infinite

\subsection{Induced Magnetic Dipole Moment}

The permeability of the dissipative medium and the conducting body is assumed to be that of free space. If the incident magnetic field is substantially uniform over the height of the conductor at a depth $d$, then the conductor will acquire an induced magnetic dipole moment, $M_{y}(d, \omega)$, which is opposite in direction to the inducing magnetic field. The dipole moment is expressed as

where

$$
M_{y}(d, \omega)=\alpha_{m} H_{y}(d, \omega),
$$

$$
\begin{aligned}
\alpha_{m} & =\text { magnetic polarizability, } \\
H_{y}(d, \omega) & =\text { incident magnetic field at depth } d .
\end{aligned}
$$

Since $M_{y}(d, \omega)$ is opposite in direction to $H_{y}(d, \omega)$, the polarizability $\alpha_{m}$ will be negative. The 
magnetic current moment, $M_{y}^{\prime}(d, \omega)$, is defined as

where

$$
M_{y}^{\prime}(d, \omega)=j \omega \mu_{0} M_{y}(d, \omega)=j \omega \mu_{0} \alpha_{m} H_{y}(d, \omega),
$$

$$
\mu_{0}=\text { permeability of free space. }
$$

The polarizabilities, $\alpha_{e}$ and $\alpha_{m}$, are functions of the volume and shape of the conductor and the relative orientation of the conductor with the inducing field. The expressions for the current moments given by (3) and (5) agree with those given by Galejs [1962] for a conducting sphere of volume $v$ if the well-known values $\alpha_{e}=3 v$ and $\alpha_{m}=-3 / 2 v$ are used for the polarizabilities.

\subsection{Perturbing Effect of Reflections}

The incident plane wave at the conductor will be perturbed by reflections of the backscattered electric and magnetic fields from the surface of the dissipative half-space. The perturbing effect of a single reflection on the electric and magnetic current moments of spheres and certain cylinders will be approximated and shown to be small.

After a single reflection from the surface of the dissipative medium, the reflected electric and magnetic fields, $E_{x r}(d, \omega)$ and $H_{y r}(d, \omega)$, respectively, induce electric and magnetic current moments in the conductor. The moments are given by

and

$$
P_{x r}^{\prime}(d, \omega)=\sigma \alpha_{e} E_{x r}(d, \omega)
$$

$$
M_{y r}^{\prime}(d, \omega)=j \omega \mu_{0} \alpha_{m} H_{y r}(d, \omega) .
$$

The reflected electric and magnetic fields in (6) and (7) may be expressed in terms of the current moments $P_{x i}^{\prime}(d, \omega)$ and $M_{y i}^{\prime}(d, \omega)$ which are induced in the conductor by the incident plane wave. Because of the large impedance mismatch at the surface of the dissipative medium, it is assumed that the backscattered electric field is totally reflected in phase and that the backscattered magnetic field is totally reflected in opposite phase. In returning to the conductor at a depth $d$, the reflected wave will have experienced a spherical spreading over the distance $2 d$. Using these approximations, the reflected fields may be obtained from the well-known propagation equations for the electromagnetic fields from electric and magnetic dipoles in an infinite dissipative medium. The electric dipole contributes to both the reflected electric and magnetic fields. Similarly, the magnetic dipole also contributes to both fields. The reflected fields are therefore written

$$
\begin{aligned}
E_{x r}(d, \omega)= & -\frac{P_{x i}^{\prime}(d, \omega)}{32 \pi \sigma d^{3}}\left(1+2 \gamma d+4 \gamma^{2} d^{2}\right) e^{-2 \gamma d} \\
& -\frac{M_{y i}^{\prime}(d, \omega)}{16 \pi d^{2}}(1+2 \gamma d) e^{-2 \gamma d}
\end{aligned}
$$

and

where

$$
\begin{aligned}
H_{y r}(d, \omega)= & -\frac{P_{x i}^{\prime}(d, \omega)}{16 \pi d^{2}}(1+2 \gamma d) e^{-2 \gamma d} \\
& +\frac{M_{y i}^{\prime}(d, \omega)}{32 \pi j \omega \mu_{0} d^{3}}\left(1+2 \gamma d+4 \gamma^{2} d^{2}\right) e^{-2 \gamma d},
\end{aligned}
$$

$$
\gamma=\left(j \omega \mu_{0} \sigma\right)^{\frac{1}{2}} \text {. }
$$

For $|\gamma d|>>1$, the exponential attenuation factors in (8) and (9) will ensure the smallness of the perturbing electric and magnetic fields. For $|\gamma d|<<1$, the perturbing moments in (6) and (7) may be expressed as

and

$$
\frac{P_{x r}^{\prime}(d, \omega)}{P_{x i}^{\prime}(d, \omega)} \approx-\frac{1}{32 \pi d^{3}}\left(\alpha_{e}+2 \gamma d \alpha_{m}\right)
$$

$$
\frac{M_{y r}^{\prime}(d, \omega)}{M_{y i}^{\prime}(d, \omega)} \approx \frac{1}{32 \pi d^{3}}\left(\alpha_{m}-2 \gamma d \alpha_{e}\right),
$$

where use is made of the expressions in (8) and (9) and of the definitions in (3) and (5). 
The ratios in (10) and (11) may readily be shown to be small for spherical conductors and for cylinders which are neither extremely needle nor extremely disk shaped. For a sphere of volume $v$ and radius $a, \alpha_{e}=3 v=4 \pi a^{3}$ and $\alpha_{m}=-\frac{1}{2} \alpha_{e}$. The ratios in (10) and (11) then become

and

$$
\frac{P_{x r}^{\prime}(d, \omega)}{P_{x i}^{\prime}(d, \omega)} \approx-\frac{1}{8}\left(\frac{a}{d}\right)^{3}(1-\gamma d)
$$

$$
\frac{M_{y r}^{\prime}(d, \omega)}{M_{y i}^{\prime}(d, \omega)}=-\frac{1}{16}\left(\frac{a}{d}\right)^{3}(1+4 \gamma d) \text {. }
$$

Since it has been assumed that $a<<d$ and that $|\gamma d|<<1$, it follows that the perturbations in the electric and magnetic moments are small for a single reflection.

The electric and magnetic polarizabilities of a right circular cylinder are given in table 1. The subscript $l$ denotes longitudinal excitation, i.e., with the particular field vector of interest along the axis of the cylinder. The subscript $t$ denotes an excitation which is transverse to the axis of the cylinder. The values in table 1 are obtained from Taylor [1960a, 1960b]. The entries for $a / b=0.1$ represent values which have been extrapolated from the results in the above references. In the dipole approximation, the dimensions of the cylinder must be much smaller than the depth at which the cylinder is embedded, so that $v<<d^{3}$. It is therefore evident from (10), (11), and table 1, that the moments due to the singly reflected electromagnetic field are small compared with the moments due to the incident plane wave for cylinders which are neither extremely needle nor extremely disk shaped.

Higher order perturbations, resulting from multiple reflections, will be even smaller than the perturbation due to a single reflection.

TABLE 1. Electric and magnetic polarizabilities of a right circular cylinder of diameter a, length $\mathrm{b}$, and volume $\mathrm{v}=\frac{\pi}{4} \mathrm{a}^{2} \mathrm{~b}$

\begin{tabular}{c|c|c|c|c}
\hline \hline$\frac{a}{b}$ & $\frac{\alpha_{e, l}}{v}$ & $\frac{\alpha_{e, t}}{v}$ & $\frac{\alpha_{m, l}}{v}$ & $\frac{\alpha_{m, t}}{v}$ \\
\cline { 2 - 4 }$\frac{1}{10}$ & 60.00 & 2.13 & -1.06 & -2.00 \\
$\frac{1}{4}$ & 15.1 & 2.32 & -1.16 & -1.85 \\
$\frac{1}{2}$ & 7.10 & 2.61 & -1.31 & -1.74 \\
1 & 3.86 & 3.17 & -1.59 & -1.58 \\
2 & 2.43 & 4.22 & -2.11 & -1.41 \\
4 & 1.75 & 6.18 & -3.09 & -1.27 \\
$\infty$ & 1.00 & $\infty$ & $-\infty$ & -1.00 \\
\hline
\end{tabular}

\section{Backscattered Electric Field at the Surface}

In this section, expressions will be derived for the backscattered electric field at the surface of the dissipative half-space for a field point directly above the conductor. The electric field contributions from the induced electric and magnetic dipoles will be treated separately and both harmonic and transient excitation will be considered.

\subsection{Electric Field From an Induced Electric Dipole}

\section{a. Harmonic Excitation}

Von Aulock [1952] has derived expressions for the electric field in a semi-infinite dissipative medium in a region directly above an embedded horizontal electric dipole. As is shown in figure 1 , the dipole is situated at the point $(0,0, d)$ and is directed along the $X$-axis. The dipole is excited at an angular frequency $\omega$. At the coordinate origin in the surface, the electric field $E_{x}^{s e}(0, \omega)$, is directed along the $X$-axis and is written

$$
E_{x}^{s e}(0, \omega)=\frac{P_{x}^{\prime}(d, \omega)}{4 \pi \sigma d^{3}}\left[\gamma^{2} d^{2} K_{2}(\gamma d)-2 e^{-\gamma d}\left(2+2 \gamma d+\gamma^{2} d^{2}\right)\right],
$$

where $\gamma=\left(j \omega \mu_{0} \sigma\right)^{\frac{1}{2}}$ and $K_{2}(\gamma d)$ is the modified Bessel function of the second kind. Using (3) 
and the plane wave relationship

$$
E_{x}(d, \omega)=\frac{\gamma}{\sigma} H_{y}(0, \omega) e^{-\gamma d}
$$

which relates the electric field incident at the conductor to the magnetic field at the surface of the half-space, (14) may be written

$$
\begin{gathered}
E_{x}^{s e}(0, \omega)=\frac{\alpha_{e}}{4 \pi d^{3}} \frac{\gamma}{\sigma} H_{y}(0, \omega) e^{-\gamma d}\left[\gamma^{2} d^{2} K_{2}(\gamma d)-2 e^{-\gamma d}\left(2+2 \gamma d+\gamma^{2} d^{2}\right)\right] . \\
\text { b. Transient Excitation }
\end{gathered}
$$

The backscattered electric field in the time domain, $\bar{E}_{x}^{e s}(0, t)$, may be expressed as a Fourier integral ${ }^{1}$ and written

$$
\bar{E}_{x}^{s e}(0, t)=\frac{1}{2 \pi} \int_{-\infty}^{\infty} E_{x}^{s e}(0, \omega) e^{j \omega t} d \omega
$$

The Fourier integral may then be transformed into a convolution integral involving the backscattered electric field response at the surface of the half-space, directly above the dipole, when a unit step of magnetic field is applied at the surface in the $y$ direction. The steps of the transformation are given in appendix 1. Equation (17) then reads

where

$$
\bar{E}_{x}^{s e}(0, t)=\int_{0}^{t} A_{x}^{e}(0, t-\lambda) H_{y}^{\prime}(0, \lambda) d \lambda,
$$

$A_{x}^{e}(0, t)=$ backscattered electric field step response at the surface from an induced electric dipole.

$H_{y}^{\prime}(0, t)=$ time derivative of the transient magnetic field applied at the surface.

The step response may explicitly be written

$$
A_{x}^{e}(0, t)=-\frac{\alpha_{e}}{4 \pi \sigma d^{4}}\left[I(\beta)+\frac{2}{(\pi \beta)^{1 / 2}}\left(2+\frac{3}{2 \beta}+\frac{1}{\beta^{2}}\right) e^{-1 / \beta}\right]
$$

where $\beta=\frac{t}{\mu_{0} \sigma d^{2}}$ and

$$
I(\beta)=\int_{0}^{\infty} \nu^{2}\left[N_{2}(\nu) \cos \nu+J_{2}(\nu) \sin \nu\right] e^{-\beta \nu^{2}} d \nu .
$$

The above expression for $I(\beta)$ is convenient for large values of $\beta$. With further manipulation, it can be shown that $I(\beta)$ may also be written

$$
I(\beta)=-\frac{1}{\pi^{1 / 2}} \int_{1}^{\infty}\left[\frac{4 \nu^{3}}{\beta^{7 / 2}}+\frac{3}{\beta^{3 / 2} \nu}+\frac{3}{\beta^{1 / 2} \nu^{3}}\right]\left(\nu^{2}-\nu\right)^{1 / 2} e^{-\frac{\nu^{2}}{\beta}} d \nu .
$$

This form is convenient for small values of $\beta$. The leading terms of an asymptotic expansion for small $\beta$ obtainable from (21) are

$$
I(\beta)=\frac{-e^{-\frac{1}{\beta}}}{2^{1 / 2} \beta^{2}}\left(1+1.68750 \beta+0.95508 \beta^{2}+0.16296 \beta^{3}-2.24885 \beta^{4}+\ldots\right) .
$$

From the exponential factor $e^{-\frac{1}{\beta}}$ in (19) and (22), it is evident that the backscattered electric field, $\bar{E}_{x}^{s e}(0, t)$, is quite small for $\mathrm{t}<<d^{2} \mu_{0} \sigma$. For large $\beta$, it can be shown that $A_{x}^{e}(0, t)$ falls off as $t^{-1 / 2}$. A graph of the dimensionless quantity $-A_{x}^{e}(0, t) \cdot \frac{4 \pi \sigma d^{4}}{\alpha_{e}}$ is shown in figure 2. The integrals were evaluated on the IBM 7090 using Gaussian integration.

\footnotetext{
${ }_{1}$ Although the Fourier integral implies high frequencies for which the displacement current in the dissipative medium becomes significant compared with the conduction current, the fields at these frequencies are severely attenuated and ccntribute negligibly except at points very near the surface.
} 


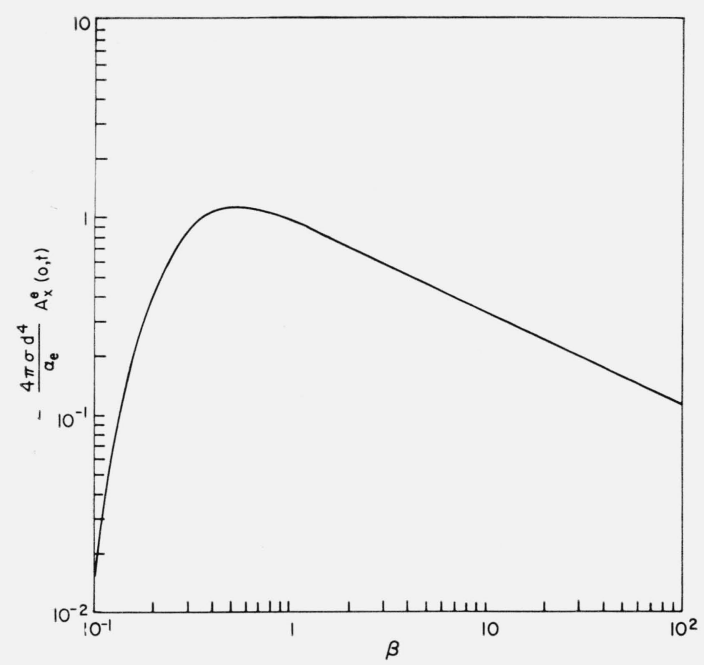

FIgURE 2. The backscattered electric field step response at the surface from an induced electric dipole.

\subsection{Electric Field From an Induced Magnetic Dipole}

\section{a. Harmonic Excitation}

Von Aulock [1952] has also derived expressions for the electric field directly above a horizontal magnetic dipole in a semi-infinite dissipative medium. As is shown in figure 1, the magnetic dipole is situated at the point $(0,0, d)$ and is directed along the $Y$-axis. The dipole is excited at an angular frequency $\omega$. At the coordinate origin in the surface, the electric field, $E_{x}^{s m}(0, \omega)$, is directed along the $X$-axis and is written

$$
E_{x}^{s m}(0, \omega)=\frac{M_{y}^{\prime}(d, \omega)}{4 \pi d^{2}}\left[\gamma d K_{1}(\gamma d)+3 K_{2}(\gamma d)-2 e^{-\gamma d}\left(\gamma d+2+\frac{3}{\gamma d}+\frac{3}{\gamma^{2} d^{2}}\right)\right]
$$

where $\gamma=\left(j \omega \mu_{0} \sigma\right)^{1 / 2}$ and $K_{1}(\gamma d), K_{2}(\gamma d)$ are the modified Bessel functions of the second kind of the first and second order, respectively. Using (5) and the plane wave relationship

equation (23) may be written

$$
H_{y}(d, \omega)=H_{y}(0, \omega) e^{-\gamma d},
$$

$$
E_{x}^{s m}(0, \omega)=\frac{j \omega \mu_{0} \alpha_{m}}{4 \pi d^{2}} H_{y}(0, \omega) e^{-\gamma d}\left[\gamma d K_{1}(\gamma d)+3 K_{2}(\gamma d)-2 e^{-\gamma d}\left(\gamma d+2+\frac{3}{\gamma d}+\frac{3}{\gamma^{2} d^{2}}\right)\right] .
$$

\section{b. Transient Excitation}

The backscattered electric field in the time domain, $\bar{E}_{x}^{s m}(0, t)$, may be expressed as a Fourier integral similar to that in (17). The Fourier integral may then be transformed into a convolution integral of the form given in (18), where $A_{x}^{m}(0, t)$ is now the backscattered electric field step response at the surface from an induced magnetic dipole. The steps of the transformation are given in appendix 2. The step response may be written explicitly as

where

$$
A_{x}^{m}(0, t)=\frac{\alpha_{m}}{4 \pi \sigma d^{4}}\left[3 I_{1}(\beta)-I_{2}(\beta)-\frac{2}{(\pi \beta)^{1 / 2}}\left(3+\frac{3}{2 \beta}+\frac{1}{\beta^{2}}\right) e^{-1 / \beta}+\frac{12}{\pi^{1 / 2}} \operatorname{erf}\left(\frac{1}{\beta^{1 / 2}}\right)\right],
$$

and

$$
\begin{gathered}
\beta=\frac{t}{\mu_{0} \sigma d^{2}}, \operatorname{erf}(x)=\int_{0}^{x} e^{-y 2} d y, \\
I_{1}(\beta)=\int_{0}^{\infty} \nu\left[N_{2}(\nu) \sin \nu-J_{2}(\nu) \cos \nu\right] e^{-\beta \nu^{2}} d \nu,
\end{gathered}
$$

$$
I_{2}(\beta)=\int_{0}^{\infty} \nu^{2}\left[N_{1}(\nu) \sin \nu-J_{1}(\nu) \cos \nu\right] e^{-\beta \nu 2} d \nu .
$$


The above expressions for $I_{1}(\beta)$ and $I_{2}(\beta)$ are convenient for large values of $\beta$. With further manipulation, it can be shown that and

$$
I_{1}(\beta)=-2+\frac{1}{4 \pi^{1 / 2}} \int_{0}^{\infty}\left[\left(\frac{8}{\beta^{3 / 2}}+\frac{14}{\beta^{1 / 2}\left(1+\nu^{2}\right)^{2}}\right) e^{-\frac{\left(1+\nu^{2}\right)^{2}}{\beta}}+\frac{20}{\left(1+\nu^{2}\right)^{3}} \operatorname{erfc}\left(\frac{1+\nu^{2}}{\beta^{1 / 2}}\right)\right] \frac{2 \nu^{2}+1}{\left(1+\nu^{2}\right)^{1 / 2}} d \nu
$$

$$
I_{2}(\beta)=-\frac{2}{\pi^{1 / 2} \beta^{5 / 2}} \int_{0}^{\infty}\left(1+\nu^{2}\right)^{3 / 2} e^{-\frac{\left(1+\nu^{2}\right)^{2}}{\beta}} d \nu
$$

where erfc $(x)=\int_{x}^{\infty} e^{-y^{2}} d y$. These forms are convenient for small values of $\beta$. The leading terms of the asymptotic expansions obtainable from (29) and (30) are

and

$$
I_{1}(\beta)=-2+\frac{e^{-1 / \beta}}{2^{1 / 2} \beta}\left(1+1.93750 \beta+0.43945 \beta^{2}-0.028687 \beta^{3}+1.97677 \beta^{4}+\ldots\right)
$$

$$
I_{2}(\beta)=-\frac{e^{-1 / \beta}}{2^{1 / 2} \beta^{2}}\left(1+0.18750 \beta-0.076172 \beta^{2}+0.10071 \beta^{3}-0.23011 \beta^{4}+\ldots\right) .
$$

It can be shown that $A_{x}^{m}(0, t)$ behaves as $e^{-1 / \beta}$ for small $\beta$ and is thus very small for $t<<d^{2} \mu_{0} \sigma$. For large $\beta$, the step response can be shown to fall off as $t^{-3 / 2}$. A graph of the dimensionless quantity $-A_{x}^{m}(0, t) \cdot \frac{4 \pi \sigma d^{4}}{\alpha_{m}}$ is shown in figure 3 . The integrals were evaluated on the IBM 7090 computer using Gaussian integration.

FiguRE 3. The backscattered electric field step response at the surface from an induced magnetic dipole.

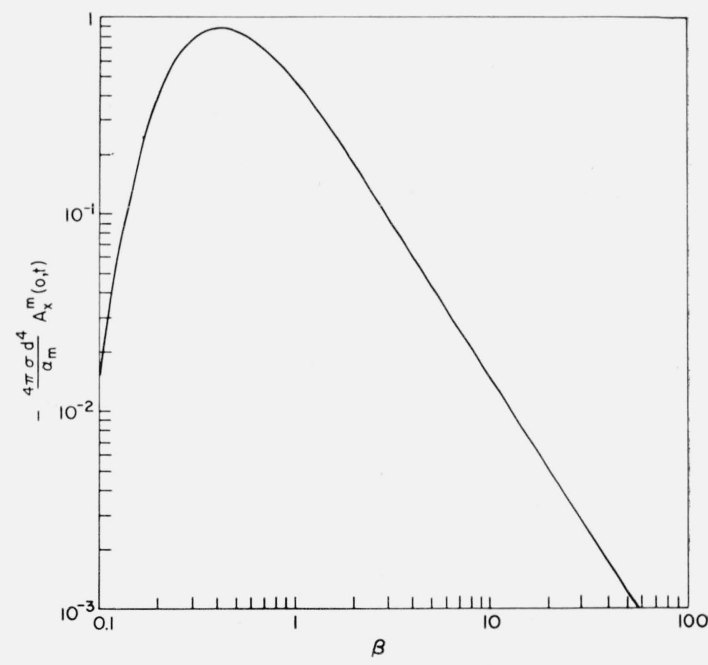

\section{Relative Importance of the Electric Field Contributions From Electric and Magnetic Dipoles}

The relative importance of the contributions by the induced electric and magnetic dipoles to the backscattered electric field at the surface will depend on the relative magnitudes of the electric and magnetic polarizabilities of the conductor. An estimate of the relative importance may be obtained by determining the ratio of the incident electric field at the surface from the magnetic dipole to that from the electric dipole. The incident electric field from the magnetic dipole, $E_{x}^{m}(0, \omega)$, is

$$
E_{x}^{m}(0, \omega)=\frac{-M_{y}^{\prime}(d, \omega)}{4 \pi^{\prime 2}}(1+\gamma d) e^{-\gamma d}
$$


and that from the electric dipole, $E_{x}^{e}(0, \omega)$, is

$$
E_{x}^{e}(0, \omega)=\frac{-P_{x}^{\prime}(d, \omega)}{4 \pi \sigma d^{3}}\left(1+\gamma d+\gamma^{2} d^{2}\right) e^{-\gamma d} .
$$

Using the definitions in (6) and (7), the ratio of the electric field contributions is written

$$
\left|\frac{E_{x}^{m}(0, \omega)}{E_{x}^{e}(0, \omega)}\right|=\left|\frac{\alpha_{m}}{\alpha_{e}} \frac{\gamma d+\gamma^{2} d^{2}}{1+\gamma d+\gamma^{2} d^{2}}\right|
$$

When $|\gamma d|<<1$, the ratio is small, provided

From (35), it is evident that

$$
\left|\frac{\alpha_{m}}{\alpha_{e}}\right|<<\left|\frac{1}{\gamma d}\right| .
$$

$$
\left|\frac{E_{x}^{m}(0, \omega)}{E_{x}^{e}(0, \omega)}\right|<\left|\frac{\alpha_{m}}{\alpha_{e}}\right|
$$

The contribution of the magnetic dipole to the electric field will, therefore, also be small when $\left|\frac{\alpha_{m}}{\alpha_{e}}\right|<<1$. This is the case for a cylinder with a small diameter to length ratio when excited longitudinally by an electric field. On the other hand, when $\left|\frac{\alpha_{m}}{\alpha_{e}}\right|$ is sufficiently large, the contribution of the magnetic dipole will be larger than that of the electric dipole. This is the case, for example, for a cylinder with a large diameter to length ratio when excited longitudinally by a magnetic field.

\section{Appendix 1}

By substituting

$$
H_{y}(0, \omega)=\int_{0}^{\infty} H_{y}(0, \lambda) e^{-j \omega \lambda} d \lambda=\frac{1}{j \omega} \int_{0}^{\infty} H_{y}^{\prime}(0, \lambda) e^{-j \omega \lambda} d \lambda
$$

in (16), the inverse Fourier transform in (17) may, upon changing the order of integration, be written

where

$$
\bar{E}_{x}^{s e}(0, t)=\int_{0}^{\infty} A_{x}^{e}(0, t-\lambda) H_{y}^{\prime}(0, \lambda) d \lambda
$$

$$
A_{x}^{e}(0, t)=\frac{\alpha_{e} \mu_{0}}{8 \pi^{2} d^{3}} \int_{-\infty}^{\infty} \frac{e^{-\gamma d}}{\gamma}\left[\gamma^{2} d^{2} K_{2}(\gamma d)-2 e^{-\gamma d}\left(2+2 \gamma d+\gamma^{2} d^{2}\right)\right] e^{j \omega t} d \omega,
$$

$\gamma=\left(j \omega \mu_{0} \sigma\right)^{1 / 2}$, and $K_{2}(\gamma d)$ is the modified Bessel function of the second kind. For convergence, $\arg \pm j \omega= \pm \frac{\pi}{2}$. Thus,

$$
A_{x}^{e}(0, t)=\frac{\alpha_{e} \mu_{0}}{4 \pi^{2} d^{3}} \operatorname{Re} \int_{0}^{\infty} \frac{e^{-\gamma d}}{\gamma}\left[\gamma^{2} d^{2} K_{2}(\gamma d)-2 e^{-\gamma d}\left(2+2 \gamma d+\gamma^{2} d^{2}\right)\right] e^{j \omega t} d \omega .
$$

When $t>0, \omega$ in (A4) is replaced by the complex variable $z=\omega+j u$, and the integration is performed around the contour shown in figure 4 . When $\epsilon \rightarrow 0$ and $R \rightarrow \infty$,

$$
A_{x}^{e}(0, t)=\frac{\alpha_{e}}{2 \pi^{2} \sigma d^{4}} \operatorname{Re} \int_{0}^{\infty} e^{-j \nu}\left[-\nu^{2} K_{2}(j \nu)-2 e^{-j \nu}\left(2+2 j \nu-\nu^{2}\right)\right] e^{-\frac{\nu^{2} t}{\mu_{0} \sigma d^{2}}} d \nu
$$

where $u=\frac{\nu^{2}}{\mu_{0} \sigma d^{2}} \cdot$ Since

$$
K_{2}(j v)=\frac{\pi}{2}\left[N_{2}(v)+j J_{2}(v)\right]
$$

where $N_{2}(\nu)$ and $J_{2}(\nu)$ are the Neumann and Bessel functions of the second order respectively, 
Figure 4. Contour in the z-plane.

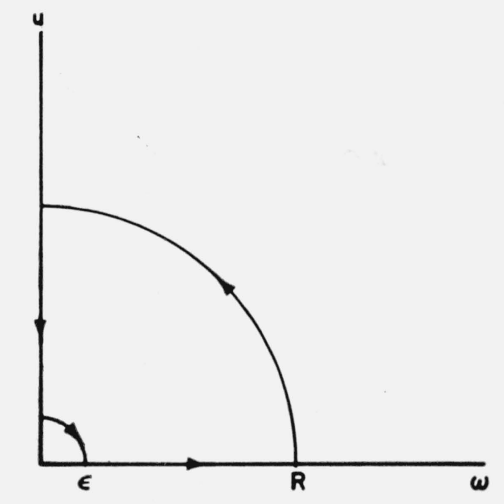

(A5) may be written

$$
\begin{aligned}
A_{x}^{e}(0, t)= & -\frac{\alpha_{e}}{4 \pi \sigma d^{4}} \int_{0}^{\infty} \nu^{2}\left[J_{2}(\nu) \sin \nu+N_{2}(\nu) \cos \nu\right] e^{-\frac{\nu t}{\mu_{0} \sigma d^{2}}} d \nu \\
& -\frac{\alpha_{e}}{\pi^{2} \sigma d^{4}} \int_{0}^{\infty}\left[\left(2-\nu^{2}\right) \cos 2 \nu+2 \nu \sin 2 \nu\right] e^{-\frac{\nu^{2} t}{\mu_{0} \sigma d^{2}}} d \nu
\end{aligned}
$$

when $t>0$. Performing some of jthe integration, (A7) is written

where $\beta=\frac{t}{\mu_{0} \sigma d^{2}}$ and

$$
A_{x}^{e}(0, t)=-\frac{\alpha_{e}}{4 \pi \sigma d^{4}}\left[I(\beta)+\frac{2}{(\pi \beta)^{1 / 2}}\left(2+\frac{3}{2 \beta}+\frac{1}{\beta^{2}}\right) e^{-1 / \beta}\right]
$$

$$
I(\beta)=\int_{0}^{\infty} \nu^{2}\left[J_{2}(\nu) \sin \nu+N_{2}(\nu) \cos \nu\right] e^{-\beta \nu^{2}} d \nu .
$$

Finally, since $A_{x}^{e}(0, t)=0$ for $t<0$,

$$
\bar{E}_{x}^{s e}(0, t)=\int_{0}^{t} A_{x}^{e}(0, t-\lambda) H_{y}^{\prime}(0, \lambda) d \lambda
$$

\section{Appendix 2}

By substituting

$$
H_{y}(0, \omega)=\int_{0}^{\infty} H_{y}(0, \lambda) e^{-j \omega \lambda} d \lambda
$$

in (25), the inverse Fourier transform may, upon changing the order of integration, be written

where

$$
\bar{E}_{x}^{s m}(0, t)=\int_{0}^{\infty} B_{x}^{m}(0, t-\lambda) H_{y}(0, \lambda) d \lambda
$$

$$
B_{x}^{m}(0, t)=\frac{\alpha_{m}}{8 \pi^{2} \sigma d^{2}} \int_{0}^{\infty} \gamma^{2} e^{-\gamma d}\left[\gamma d K_{1}(\gamma d)+3 K_{2}(\gamma d)-2 e^{-\gamma d}\left(\gamma d+2+\frac{3}{\gamma d}+\frac{3}{\gamma^{2} d^{2}}\right)\right] e^{j \omega t} d \omega
$$

$\gamma=\left(j \omega \mu_{0} \sigma\right)^{1 / 2}$, and $K_{1}(\gamma d)$ and $K_{2}(\gamma d)$ are the modified Bessel functions of the second kind of the first and second order, respectively. Noting that $\arg \pm . j \omega= \pm \pi / 2$, (A13) may be written

$$
B_{x}^{m}(0, t)=\frac{\alpha_{m}}{4 \pi^{2} \sigma d^{2}} \operatorname{Re} \int_{0}^{\infty} \gamma^{2} e^{-\gamma d}\left[\gamma d K_{1}(\gamma d)+3 K_{2}(\gamma d)-2 e^{-\gamma d}\left(\gamma d+2+\frac{3}{\gamma d}+\frac{3}{\gamma^{2} d^{2}}\right)\right] e^{j \omega t} d \omega .
$$


When $t<0$, the impulse response, $B_{x}^{m}(0, t)$, is equal to zero since it is assumed that $H_{y}(0, t)=0$ for $t \leqq 0$. Integrating (A12) by parts, it follows that

where

$$
\bar{E}_{x}^{s m}(0, t)=\int_{0}^{t} A_{x}^{m}(0, t-\lambda) H_{y}^{\prime}(0, \lambda) d \lambda
$$

$$
A_{x}^{m}(0, t)=\frac{\mu_{0} \alpha_{m}}{4 \pi^{2} d^{2}} \operatorname{Re} \int_{0}^{\infty} e^{-\gamma d}\left[\gamma d K_{1}(\gamma d)+3 K_{\varepsilon}(\gamma d)-2 e^{-\gamma d}\left(\gamma d+2+\frac{3}{\gamma d}+\frac{3}{\gamma^{2} d^{2}}\right)\right] e^{j \omega t} d \omega .
$$

When $t>0, \omega$ in (A16) is replaced by the complex variable $z=\omega+j u$, and the integration is performed around the contour shown in figure 4. When $\epsilon \rightarrow 0$ and $R \rightarrow \infty$,

$$
A_{x}^{m}(0, t)=\frac{\alpha_{m}}{2 \pi^{2} \sigma d^{4}} \operatorname{Re} j \int_{0}^{\infty} \nu e^{-j \nu}\left[j \nu K_{1}(j \nu)+3 K_{2}(j \nu)-2 e^{-j \nu}\left(j \nu+2+\frac{3}{j \nu}-\frac{3}{\nu^{2}}\right)\right] e^{-\frac{\nu^{2} t}{\mu_{0 \sigma d^{2}}} d \nu},
$$

where $u=\frac{\nu^{2}}{\mu_{0} \sigma d^{2}}$. Since

$$
K_{1}(j \nu)=-\frac{\pi}{2}\left[J_{1}(\nu)-j N_{1}(\nu)\right]
$$

and

$$
K_{2}(j v)=\frac{\pi}{2}\left[N_{2}(v)+j J_{2}(\nu)\right]
$$

where $J_{2}(\nu)$ and $N_{2}(\nu)$ are the Bessel and Neumann functions of the second order respectively, (A17) may be written

$$
\begin{array}{r}
A_{x}^{m}(0, t)=\frac{\alpha_{m}}{4 \pi \sigma d^{4}} \int_{0}^{\infty}\left\{3 \nu\left[N_{2}(\nu) \sin \nu-J_{2}(\nu) \cos \nu\right]-\nu^{2}\left[N_{1}(\nu) \sin \nu-J_{1}(\nu) \cos \nu\right]\right. \\
\left.-\frac{4}{\pi}\left[\left(2 \nu-\frac{3}{\nu}\right) \sin 2 \nu-\left(\nu^{2}-3\right) \cos 2 \nu\right]\right\} e^{-\frac{\nu^{2} t}{\mu_{0} \sigma d^{2}}} d \nu .
\end{array}
$$

Performing some of the integration, (A20) is written

$$
A_{x}^{m}(0, t)=\frac{\alpha_{m}}{4 \pi \sigma d^{4}}\left[3 I_{1}(\beta)-I_{2}(\beta)-\frac{2}{(\pi \beta)^{1 / 2}}\left(3+\frac{3}{2 \beta}+\frac{1}{\beta^{2}}\right) e^{-1 / \beta}+\frac{12}{\pi^{1 / 2}} \operatorname{erf}\left(\frac{1}{\beta^{1 / 2}}\right)\right],
$$

where

$$
\begin{gathered}
\beta=\frac{t}{\mu_{0} \sigma d^{2}}, \quad \operatorname{erf}(x)=\int_{0}^{x} e^{-i^{2}} d t, \\
I_{1}(\beta)=\int_{0}^{\infty} \nu\left[N_{2}(\nu) \sin \nu-J_{2}(\nu) \cos \nu\right] e^{-\beta \nu^{2}} d \nu,
\end{gathered}
$$

and

$$
I_{2}(\beta)=\int_{0}^{\infty} \nu^{2}\left[N_{1}(\nu) \sin \nu-J_{1}(\nu) \cos \nu\right] e^{-\beta \nu^{2}} d \nu
$$

The author is grateful to Dr. A. H. Van Tuyl of the U.S. Naval Ordnance Laboratory for transforming the Fourier integrals into convolution integrals and for the analysis in preparation for the programing of the step responses for the IBM 7090 computer. 


\section{References}

Galejs, J. (Sept.-Oct. 1962), Scattering from a conducting sphere embedded in a semi-infinite dissipative medium, J. Research NBS 66D (Radio Prop.) No. 5, 607-612.

Negi, J. G. (June 1962), Inhomogeneous cylindrical ore body in presence of a time varying magnetic field, Geophysics XXVII, No. 3, 386-392.

Stratton, J. A. (1941), Electromagnetic theory, ch. IX, 563-573 (McGraw-Hill Book Co., Inc., New York, N.Y.).

Taylor, T. T. (July-Sept. 1960a), Electric polarizability of a short right circular conducting cylinder, J. Research NBS 64B (Math. and Math. Phys.) No. 3, 135-143.

Taylor, T. T. (Oct.-Dec. 1960b), Magnetic polarizability of a short right circular conducting cylinder, J. Research NBS 64B (Math. and Math. Phys.) No. 4, 199-210.

Von Aulock, W. (1952), Low frequency electromagnetic dipole fields in a semi-infinite conductor, U.S. Navy Dept., BuShips Minesweeping Sec., Tech. Rept. No. 104.

Wait, James R. (Oct. 1951), A conducting sphere in a time varying magnetic field, Geophysics XVI, $666-672$.

Wait, James R. (June 1960), On the electromagnetic response of a conducting sphere to a dipole, Geophysics $\mathbf{X X V}$, No. 3, 649-658.

(Paper 67D4-278) 